\title{
A new hypnic paradigm of neurodegenerative proteinopathies
}

\author{
Jason D Warren, Camilla N Clark \\ Dementia Research Centre, UCL Institute of Neurology, University College London \\ London, United Kingdom
}
Correspondence: $\quad$ Prof. Jason Warren Dementia Research Centre UCL Institute of Neurology University College London London WC1N 3BG
Email: jason.warren@ucl.ac.uk
Tel: $+44[0] 2034484773$
Fax: +44 [0]2034483104

Key words: sleep; dreams; dementia; frontotemporal dementia; Alzheimer's disease Word count: main text 667 
Sleep is a major emerging area of interest in neurodegenerative diseases. A compelling case has been made that sleep disruption contributes to the pathogenesis of Alzheimer's disease (AD)[1]. In this paper we draw attention to mounting recent evidence that disordered sleep is a feature of diverse neurodegenerative diseases besides $\mathrm{AD}$, and may differentiate these diseases on clinical, electrophysiological and neurohormonal grounds[2-8]. In addition to well-recognised sleep phenotypes of Parkinson's and prion disease, clinical sleep disturbance and disordered sleep architecture are increasingly reported in the frontotemporal dementia (FTD) spectrum[2,3].

FTD is an informative test case that assesses the wider pathophysiological relevance of sleep in proteinopathies. Clinically, patients with FTD commonly develop excessive somnolence as well as narcolepsy-like attacks, insomnia and other sleep-related symptoms[2,4]; sleep disturbance may be more prevalent in FTD than in $\mathrm{AD}[4]$ and forms part of a broader repertoire of chronobiological and autonomic symptoms[4]. FTD is associated electrophysiologically, with disruption of macro- and micro-structural parameters that may be more severe and occur earlier than in $\mathrm{AD}[5]$. AD and FTD show divergent profiles of hypothalamic neuropeptide alterations; whereas $\mathrm{AD}$ may be uniquely associated with elevated orexin levels[1], FTD is associated with reduced plasma and CSF orexin that correlates with excessive daytime somnolence[2,3]. In addition to differentiating AD from non-AD (FTD) pathologies, sleep phenotype may further stratify proteinopathies within the FTD spectrum, as exemplified by genetically-mediated forms of FTD. Mutation of the MAPT gene has been linked to severe insomnia, sleep fragmentation, reduced REM latency and slowwave sleep while mutation of the C9orf72 gene may be associated with REM-behaviour disorder [6-8], corroborated in mouse models[9]. 
We propose that sleep-related processes are integral to the pathogenesis of neurodegenerative diseases. Sleep may translate the effects of pathogenic protein spread in vulnerable neuronal populations to the large-scale neural ensembles that drive clinical phenotypes. We outline this idea in Figure 1. Because neurodegenerative proteinopathies preferentially target particular neural systems (at least earlier in the course of disease) and these same systems are often integrally involved in circadian cycling, the pathophysiological effects of sleep disturbance - and more specifically, impairment of restorative or reparative sleep processes acting at synaptic level - will tend to be focused within the vulnerable circuitry that triggers the sleep derangement[1,10]. This would create a substrate for vicious cycling, with amplification of dysfunction leading ultimately to cell death - and further sleep disruption. If this idea is correct, sleep disturbance is a potential driver of neurodegeneration in a range of proteinopathies.

We assert that further work to assess the implications of disordered sleep in dementia is urgently required, and this should entail a broadening of vision beyond $\mathrm{AD}$ to other neurodegenerative diseases whose hypnic phenotypes have not yet been fully characterised. There is a need to establish the relation of sleep disruption to other disease markers and to assess the extent to which sleep phenotype may predict the evolution of neurodegeneration across proteinopathies (and might differentiate particular proteinopathies). This will entail head-to-head comparison of diseases with molecular correlation in longitudinal natural history studies and the further development of animal models and computational approaches that will allow sleep parameters to be directly manipulated. Because sleep disturbance is so common in the population at large, identification of a useful hypnic biomarker of neurodegeneration may require isolation of a disease-specific microarchitectural feature of the sleep phenotype or a feature that (while not specific) indexes disease progression in the clinical context. A number 
of vistas of sleep physiology remain under-explored in neurodegenerative populations but might be anticipated on clinical grounds to yield novel insights into particular diseases. One example is the cognitive neuropsychology and functional neuroanatomy of dreaming: dreams may sensitively signal dysfunction of the large-scale neural networks that mediate sleep cycle transitions and may be particularly vulnerable to loss of visuospatial imagery in AD and its variant phenotypes, as well as to semantic and affective disintegration in syndromes of FTD. Besides the prospect of new clinical and laboratory biomarkers, defining hypnic mechanisms of proteinopathies promises to yield a powerful new pathophysiological paradigm of neurodegenerative disease.

\section{Acknowledgments}

The Dementia Research Centre is supported by Alzheimer's Research UK, the Brain Research Trust and the Wolfson Foundation. This work was funded by the Wellcome Trust, the UK Medical Research Council and the NIHR Queen Square Dementia Biomedical Research Unit. CNC was supported by The National Brain Appeal - Frontotemporal Dementia Research Fund. JDW was supported by a Wellcome Trust Senior Clinical Fellowship [Grant No 091673/Z/10/Z]. 


\section{References}

1 Mander, B.A., Winer, J.R., Jagust, W.J., and Walker, MP. (2016) Sleep: a novel mechanistic pathway, biomarker, and treatment target in the pathology of Alzheimer's disease? Trends Neurosci. 39, 552-66.

2 Çoban, A., et al. (2013) Reduced orexin-A levels in frontotemporal dementia: possible association with sleep disturbance. Am J Alzheimers Dis Other Demen. 28, 606-11.

3 Liguori, C., et al. (2014) Cerebrospinal-fluid orexin levels and daytime somnolence in frontotemporal dementia. J Neurol. 261, 1832-6.

4 Ahmed, R.M, Iodice, V., Daveson, N., Kiernan, M.C., Piguet, O., and Hodges, J.R. (2015) Autonomic dysregulation in frontotemporal dementia. J Neurol Neurosurg Psychiatry. 86, 1048-9.

5 Bonakis, A., et al. (2014) Sleep in frontotemporal dementia is equally or possibly more disrupted, and at an earlier stage, when compared to sleep in Alzheimer's disease. J Alzheimers Dis. 38, 85-91.

6 Gemignani, A., et al. (2005) Slow wave and REM sleep mechanisms are differently altered in hereditary Pick disease associated with the TAU G389R mutation. Arch Ital Biol. 143, 65-79.

7 Spector, A.R., et al. (2011) Anatomy of disturbed sleep in pallido-ponto-nigral degeneration. Ann Neurol. 69, 1014-25.

8 Daoud, H., et al. (2014) C9orf72 repeat expansions in rapid eye movement sleep behaviour disorder. Can J Neurol Sci. 41, 759-62.

9 Koss, D.J., et al. (2016) Mutant Tau knock-in mice display frontotemporal dementia relevant behaviour and histopathology. Neurobiol Dis. 91, 105-23.

10 Sakurai, T. and Mieda, M. (2011) Connectomics of orexin-producing neurons: interface of systems of emotion, energy homeostasis and arousal. Trends Pharm Sci 32, 451-462. 


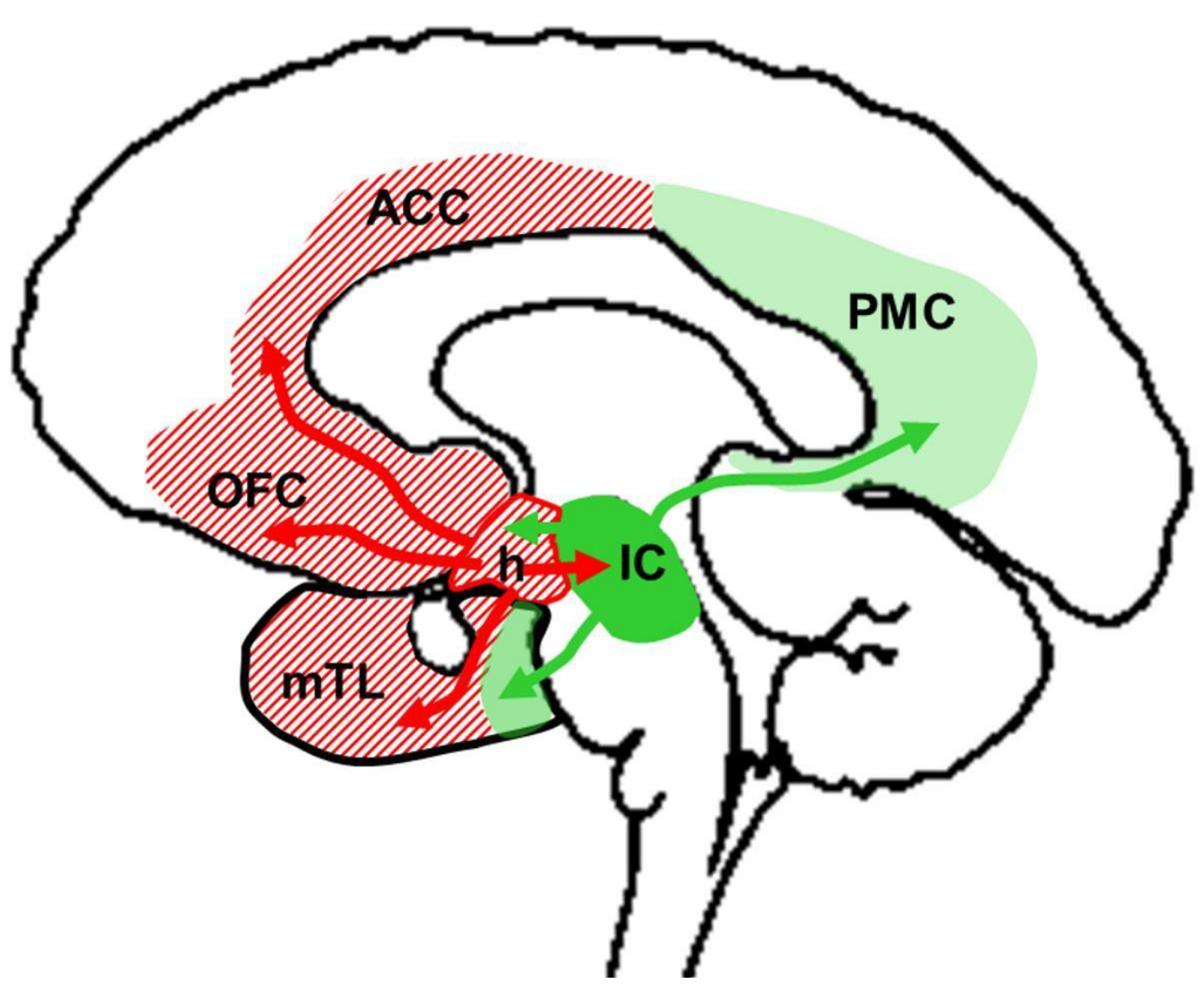

This cartoon of a mid-sagittal brain projection schematises the pathophysiological interaction of sleep-related neural circuitry with distributed networks targeted by Alzheimer's disease (green) and non-Alzheimer proteinopathies in the frontotemporal dementia spectrum (red cross-hatched), in line with recent clinical and neurohormonal evidence [2-5,10]. In Alzheimer's disease, cholinergic and other brainstem projection pathways in the isodendritic core (IC) preferentially target postero-medial cortex (PMC) and medial temporal lobe (mTL) structures including entorhinal cortex and hippocampus, while FTD proteinopathies preferentially target anterior cingulate cortex (ACC), basal orbitofrontal cortex (OFC) and anterior MTL structures connected to hypothalamus $(\mathbf{h})$. Each of these proteinopathies disrupts interactions between brainstem, basal forebrain and hypothalamic cell populations involved in sleep and circadian cycling (bidirectional arrows): in Alzheimer's disease, this may lead initially to compensatory increases in orexinergic activity with prominent insomnia, while in proteinopathies causing frontotemporal dementia, primary degeneration of orexinergic pathways may lead to excessive somnolence. In each case, loss of the restorative function of normal sleep focuses synaptic dysfunction in the projection zones targeted by the proteinopathy (long arrows), establishing a vicious cycle: sleep disturbance begets neurodegenerative damage which in turn begets further sleep disturbance. Both frontotemporal dementia and Alzheimer's disease show considerable clinical heterogeneity and uniform sleep phenotypes of these diseases are therefore unlikely a priori. However, this highly over-simplified scheme predicts that sleep disturbance is a driver of the pathological process across neurodegenerative proteinopathies while specific sleep phenotypes may distinguish proteinopathies. 